\title{
V Appliance- Versatility for Enhanced Applicability
}

\section{Mithun K Naik ${ }^{1}$, Jayakrishnan ${ }^{2 *}$, Sharmila K Shetty ${ }^{3}$, K Nillan Shetty and Rohan Rai $^{5}$}

${ }^{1}$ Assistant Professor, Department of Orthodontics and Dentofacial Orthopedics, AJ

Institute of Dental Sciences, Mangalore, India

${ }^{2}$ Practicing Orthodontist, Danthashreya Multispeciality Dental Clinic,

Thiruvananthapuram, Kerala, India

${ }^{3}$ Practicing Orthodontist, Durga Daya Dental Clinic and Implant Center, Mangalore, India

${ }^{4}$ Professor and Head, Department of Orthodontics and Dentofacial Orthopedics, $A J$

Institute of Dental Sciences, Mangalore, India

${ }^{5}$ Professor, Department of Orthodontics and Dentofacial Orthopedics, AJ Institute of

Dental Sciences, Mangalore, India

*Corresponding Author: Jayakrishnan U, Practising Orthodontist, Danthashreya

Multispeciality Dental Clinic, Thiruvananthapuram, Kerala, India.
Received: November 26, 2021

Published: February 21, 2022

(C) All rights are reserved by Jayakrishnan U., et al.

\begin{abstract}
Introduction: In the modern era, where the treatment mechanics are being refined to suit the requirements of the patient and ease of application by the clinician, there is an increasing demand for versatility in the appliance being used. Complexity doesn't contribute to better patient compliance or help the clinician in carrying out the mechanics with ease. Patients are demanding minimal invasive treatment to correct the malocclusions. There is need for innovations in appliance design to customise it according to the patient needs. Description: Minor tooth corrections can be brought about by removable appliances and has a better compliance factor with the patient. The complexity of the appliance and the treatment duration might hinder the patient from approaching a clinician for correction of minor problems relating the dentition. In the present article, a new appliance is made to overcome the problems with the present systems in dealing with minor tooth corrections and it can be modified according to the requirements of the corrections intended to achieve. Results: The objective of the treatment could be achieved with the use of the appliance and patient was fully satisfied with the treatment results.
\end{abstract}

Keywords: Diastema Closure; Minor Tooth Corrections; Versatility

\section{Introduction}

Addressing the chief problem with which an individual approach a clinician is of prime importance in gaining the confidence of the patient. At times, we tend to overlook it, due to the sophisticated mechanics we use, failing to convince the patient on the ultimate treatment results [1-5]. Minor tooth corrections should be dealt in such a way that the patient feels the main problem is addressed without much taxing on the other structures of the oral cavity, be it with other teeth. With fixed appliances, we tend to increase the complexity of the treatment and also duration of the whole treatment extends.

There are many methods at present to address minor dental problems such as midline diastema, single tooth rotations, spac- 
ing between the teeth, minor sagittal corrections and others [67]. Most of the techniques deals with comprehensive treatment of the dental arches, keeping in mind the stability of the results to be achieved. But in the due course, we tend to miss out on the patient compliance and confidence and often the treatment is further delayed. The most critical problem with correction of spacings, be it with the midline diastema, is the relapse rate. Even after extensive treatment with fixed appliance therapy, a fixed retainer needs to be given. This leads to reduced motivation among patients to proceed with a full-fledged fixed orthodontic therapy.

In order to address these issues, the present article introduces a new removable appliance that can be used to correct minor tooth rotations, spacing in the dentition and midline diastemas without any complex treatment procedures.

\section{Appliance design}

This versatile appliance or the ' $\mathrm{V}$ ' appliance is composed of (i) a Serpentine hook made of 0.9 stainless steel wire (Figure 1a-e) from the acrylic plate in the palate, extending between the first premolar and canine projecting at midline of the long axis of canine (ii) triangular clasp is placed in between maxillary premolars \& ball end clasp on mandibular premolars (iii) Adam's clasp on the molars for retention (iv) elastics for attaching between the Serpentine hooks of both sides (Figure 2) (v) acrylic plate on the palatal/lingual side to act as a connector and to stabilize the appliance. Flowable composite resin can be used of the labial surfaces of teeth to secure the elastics onto the surface.

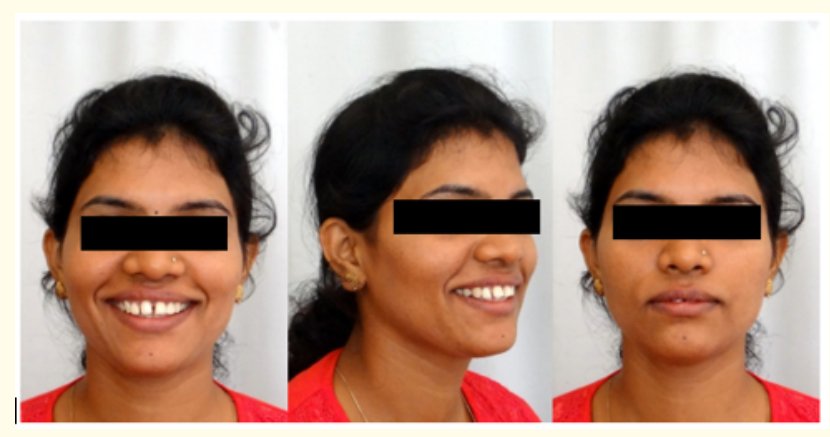

Figure 1: Extra oral photographs.

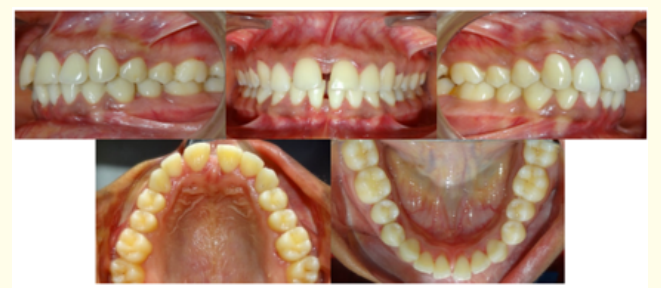

Figure 2: Intra Oral Photographs.

It can be used in both the maxillary and mandibular arches without any constructional modifications. It has the advantage of being a removable appliance so that the patient can remove it whenever they feel at social appearances, so that the compliance increases.

\section{Biomechanics of ' $V$ ' appliance}

Effective correction of various malocclusion carried out by the ' $V$ ' Appliance is illustrated with the help of diagrams. It proves to be effective in correcting various common malocclusions without the intervention with a fixed appliance and is very useful in compliant patients. It can be given in Deep bite (Figure 3), Openbite (Figure 4), Diastema closure (Figure 5) and even for intrusion (Figure 6) and extrusion (Figure 7) of the teeth to an extent.

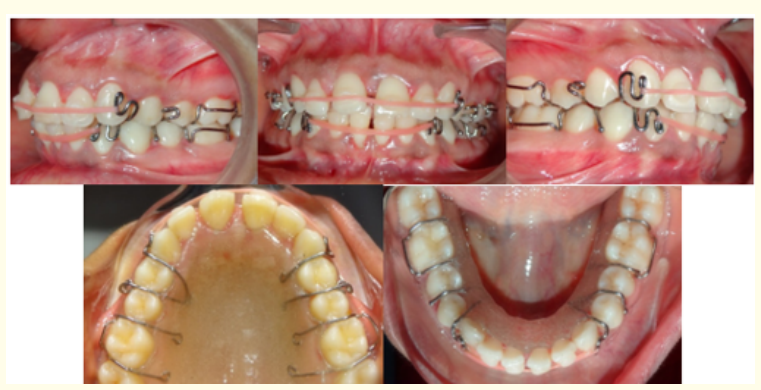

Figure 3: Intra Oral Photographs with the 'V' Appliance.

\section{Case Report}

- Introduction: A 21-year-old female patient reported to our clinic with a chief complaint of spacing between the upper teeth. Extra oral examination from the frontal view of the patient revealed symmetry both in transverse and vertical dimensions (Figure 8). 

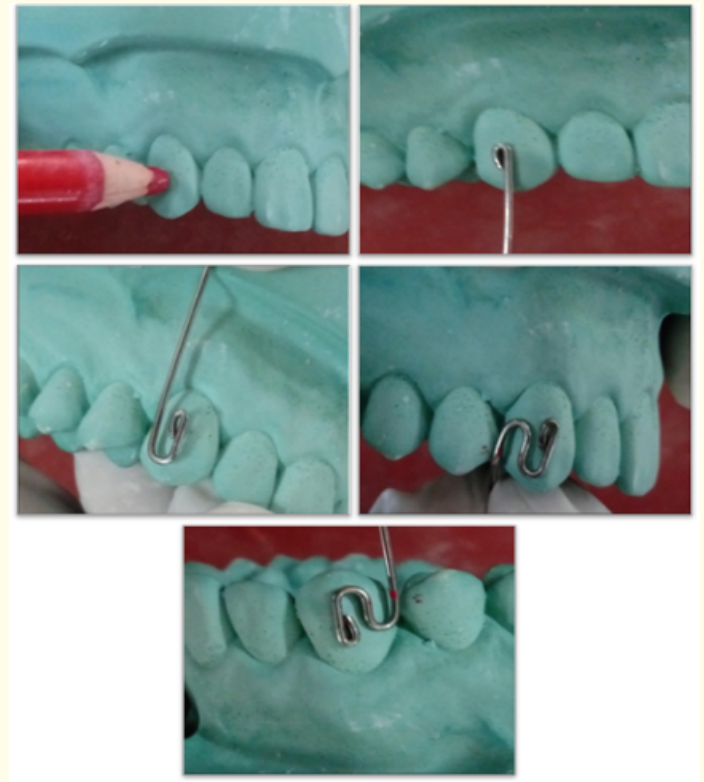

Figure 4a-e: Steps in fabrication of Serpentine hooks.

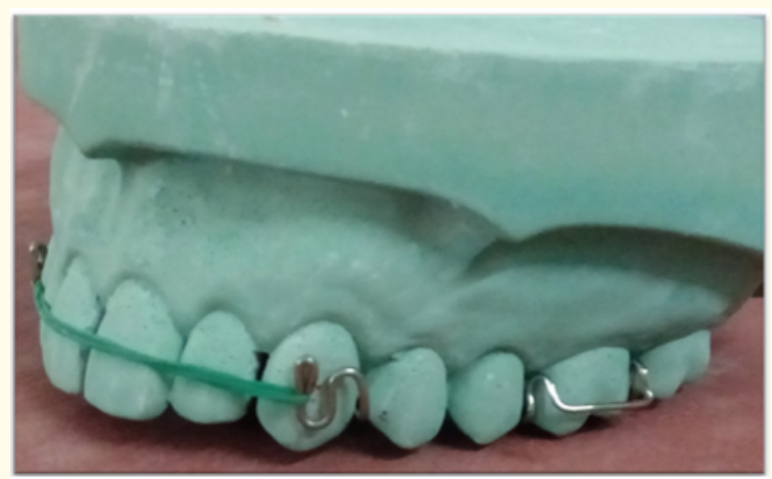

Figure 5: Elastics connecting the two Serpentine hooks.

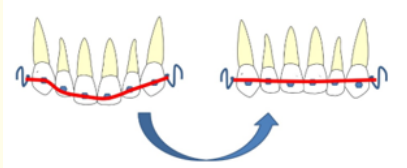

Figure 6: Deep bite correction

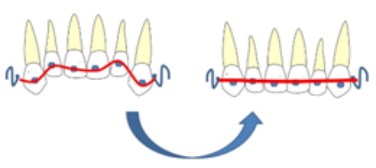

Figure 7: Open bite correction
- Diagnosis: However, the patient presented with a midline diastema of $3 \mathrm{~mm}$. Profile view showed a convex profile with normal lip fullness. Soft tissue chin thickness was normal. Intra oral examination showed presence of all compliments of teeth till the $2^{\text {nd }}$ molars. She presented with Angle's Class I malocclusion with mild crowding in the lower arch and midline diastema (Figure 9). There was no evidence of any midline shift.

- Treatment plan: Fixed appliance therapy was indicated for the patient and suggested to the patient, but she was reluctant to go for a Fixed Orthodontic treatment. Patient was willing for the treatment with a removable appliance. Therefore, a second treatment option in the form of ' $\mathrm{V}$ ' Appliance was suggested (Figure 10).

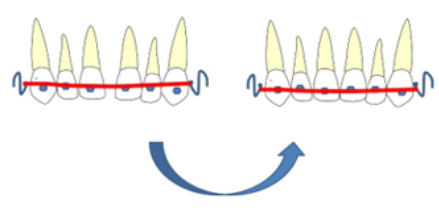

Figure 8: Diastema correction.

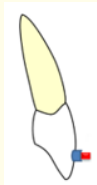

Figure 9: Intrusion.

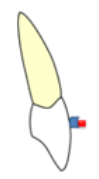

Figure 10: Extrusion.

' $\mathrm{V}$ ' Appliance was used and mild force was applied initially to get the midline diastema closed and also to correct the mild crowding in the lower arch. The transverse and vertical dimensions was maintained due to the advantage of versatility of the appliance to apply force magnitude in desired directions. 
The pre-treatment and post-treatment photographs (Figure 11) revealed successful closure of the midline diastema with the transverse and vertical dimension maintained and bilateral Class I Molar and Canine relationship remained unchanged.

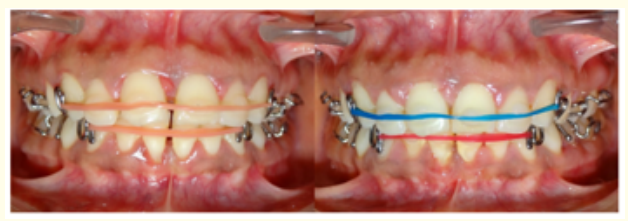

Figure 11: Pre-treatment and Post-treatment Photographs with the $\mathrm{V}$ appliance.

\section{Discussion}

The key feature of this appliance is its ease of fabrication and the reduced treatment time to close the spaces between the dentitions. Since extensive therapy is not being carried out, patient compliance to this appliance seems to be better and the patient is better motivated. Conventional treatment methods comprise of extensive treatment for a longer duration, which might not be accepted by the patient.

The follow up studies of the cases treated with the appliance shows that even if ideal occlusion and dental alignment has been achieved, a fixed retainer would be ideal to prevent the relapse [8]. Preserving the treatment results achieved can contribute to a higher level of patient satisfaction. Although the outcome of treatment with the appliance has been found to be effective, one of the drawbacks associated with use of a removable appliance of this kind is the inability of the appliance to bring about any tip to the anterior teeth. This in turn prevents the correction of mesiodistal inclination of the teeth.

Since the appliance depends on patient compliance, it is imperative that the patient is motivated to use the appliance regularly until the desired treatment result is achieved.

It also possesses an inherent drawback of inability to effect multiple tooth movements. Considering the treatment results that is achieved with the appliance, the benefits outweigh the disadvantages when ideal case selection is done.

\section{Conclusion}

The ' $V$ ' appliance serves the purpose of correcting minor tooth rotations and spacing within a reduced span of time, attracting more patients to take up the treatment with the appliance. Patient motivation seems to be good, with the treatment results in short durations. Relapse tendencies seem to be unavoidable, so a fixed retainer would be best to sustain the results achieved. The simplicity in design, the ease of fabrication of appliance, an increased patient compliance and the versatility of the appliance to deal with malocclusions tends to give the best treatment results

\section{Conflict of Interest}

None.

\section{Source of Support}

Nil.

\section{Bibliography}

1. Ardeshna AP., et al. "The effect of rewards on orthodontic patient compliance". Journal of Indian Orthodontic Society 52 (2018): 198-203.

2. Beckwith FR., et al. "An evaluation of factors affecting duration of orthodontic treatment". American Journal of Orthodontics and Dentofacial Orthopedics 115 (1999): 439-447.

3. Mavreas D and Athanasiou AE. "Factors affecting the duration of orthodontic treatment: A systematic review". European Journal of Orthodontics 30 (2008): 386-395.

4. Starnbach HK and Kaplan A. "Profile of an excellent orthodontic patient”. The Angle Orthodontist 45 (1975): 141-145.

5. Skidmore KJ., et al. "Factors influencing treatment time in orthodontic patients". American Journal of Orthodontics and Dentofacial Orthopedics 129 (2006): 230-238.

6. Gleghorn T. "Direct composite technique for a smile makeover". Dental Today 16 (1997): 40-44.

7. Munshi A and Munshi AK. "Midline space closure in the mixed dentition: A case report". Journal of Indian Society of Pedodontics and Preventive Dentistry 19 (2001): 57-60. 
8. Follin ME. "Orthodontic movement of maxillary incisor into the midline. A case report". The Swedish Dental Journal 9 (1985): 9-13.

\section{Assets from publication with us}

- Prompt Acknowledgement after receiving the article

- Thorough Double blinded peer review

- Rapid Publication

- Issue of Publication Certificate

- High visibility of your Published work

Website: www.actascientific.com/

Submit Article: www.actascientific.com/submission.php

Email us: editor@actascientific.com

Contact us: +919182824667 